\title{
Surgical management of metastatic adrenal tumors: Decision-making factors in imaging
}

\author{
SUNAO SHOJI $^{1}$, YUKIO USUI ${ }^{1}$, MAYURA NAKANO ${ }^{1}$, KAZUYA HANAI ${ }^{1}$, \\ HARUHIRO SATO $^{2}$, TOYOAKI UCHIDA ${ }^{1}$ and TOSHIRO TERACHI ${ }^{1}$
}

\author{
Departments of ${ }^{1}$ Urology, and ${ }^{2}$ Medicine, Tokai University School of Medicine, Tokyo 192-0032, Japan
}

Received June 22, 2010; Accepted August 27, 2010

DOI: $10.3892 / \mathrm{ol} .2010 .183$

\begin{abstract}
The present study aimed to clarify decision-making factors based on imaging for laparoscopic adrenalectomy (LA) or open adrenalectomy (OA) for adrenal metastasis (AM) based on our previous experience. From November 2003 to November 2006, 11 adrenalectomies were performed for AM for malignancies such as lung cancer, renal cell carcinoma (RCC) and breast cancer at Tokai University Hospital. A diagnosis of AM for these malignancies was suspected whenever a newly diagnosed adrenal mass was located, characterized by a basal computed tomography (CT) density superior to 10 Hounsfield units, strong or heterogeneous vascular enhancement following contrast injection and/or increasing size in sequential imaging studies. There was no evidence of extra-AM. The approach to surgical management using LA or OA was determined on the basis of CT and/or magnetic resonance imaging. The patients were reviewed every 2 or 3 months by physical examination and systemic CT. We analyzed the decision-making factors based on imaging for surgical management with LA or OA from the results of oncological outcome, imaging, intraoperative and pathohistological findings. In this study, 9 patients underwent 11 adrenalectomies ( 9 laparoscopic and 2 open procedures). Non-small cell lung cancer was the most common primary malignancy (5 adrenalectomies of 4 patients), followed by RCC ( 4 adrenalectomies of 4 patients) and breast cancer ( 2 adrenalectomies of 1 patient). The median tumor size for the LA group was $3.1 \pm 0.7 \mathrm{~cm}$ (range 2.1-4.3) and for the OA group, $6.1 \pm 0.8 \mathrm{~cm}(5.5$ and $6.7 \mathrm{~cm})(\mathrm{p}=0.001)$. The operative time for the LA group was $127 \pm 42 \mathrm{~min}$ (range 90-215) and for the OA group, $224 \pm 47 \mathrm{~min}(190$ and $257 \mathrm{~min})(\mathrm{p}=0.018)$. Blood
\end{abstract}

Correspondence to: Dr Sunao Shoji, Department of Urology, Tokai University Hachioji Hospital, Ishikawa-machi 1838, Hachioji, Tokyo 192-0032, Japan

E-mail: sunashoj@mail.goo.ne.jp

Abbreviations: AM, adrenal metastasis; LA, laparoscopic adrenalectomy; OA, open adrenalectomy; CT, computed tomography; MRI, magnetic resonance imaging; HU, Hounsfield units; RCC, renal cell carcinoma; NSCLC, non-small cell lung cancer; MODFS, median overall disease-free survival; DFSR, disease-free survival rate

Key words: adrenal metastasis, surgical management, imaging study loss for the LA group was $49 \pm 63 \mathrm{~g}$ (range 3-207) and for the OA group, $340 \pm 10 \mathrm{~g}$ (333 and $347 \mathrm{~g})(\mathrm{p}<0.001)$. No complications were noted and no conversion of LA to OA occurred. All 9 adrenal tumors selected for LA were removed safely without strong adhesion to the surrounding tissue. Two adrenal tumors removed by OA had a strong adhesion to the surrounding tissue. All 9 patients had complete resection, without capsular disruption and a negative margin in the pathological findings. No port-site and local recurrences occurred. No patients presented with local relapse or port-site metastasis. Disease-free survival rate for the LA group was $57 \%$ and for the OA group, $50 \%$ $(\mathrm{p}=0.661)$. LA is a less invasive treatment than OA for AM. However, for complete resection, OA should be selected for cases where resection by LA is difficult. Therefore, in the decision making towards the appropriate surgical management with LA or OA, it is important to closely assess pre-operative imaging. Imaging features supporting OA include no detection of fatty tissue between the tumor and proximal organs, tumors with an irregular contour, large tumors and tumors with a cystic component.

\section{Introduction}

Since Gagner et al performed the first resection of an adrenal tumor by laparoscopic adrenalectomy (LA) in 1992 (1), it has become the standard treatment for benign adrenal tumors and has markedly reduced the morbidity associated with this operation. A comparison of traditional open adrenalectomy (OA) to LA showed that the length of hospital stay and the use of post-operative analgesics in LA are decreased, and the rate of return to normal activities is increased.

Strong et al were able to more definitively compare the results of LA vs. OA at the Memorial Sloan-Kettering Cancer Center (2). These authors showed that LA compared to OA resulted in less morbidity and achieved similar oncological outcomes. However, applying LA for solitary metastasis or primary adrenal carcinoma remains a matter of considerable controversy, since port-site metastasis and dissemination have been reported when using LA to treat adrenal malignancies. Port-site metastasis or dissemination without suspicion of incision or injury to the tumor has yet to be reported. Therefore, it is important to ensure appropriate surgical management to prevent incision or injury to the tumor in adrenal malignancy when deciding whether to apply LA or OA. 
Table I. Patient data and characteristics of the primary malignancy.

\begin{tabular}{|c|c|c|c|c|c|}
\hline \multirow[b]{2}{*}{ Patient } & \multirow{2}{*}{$\begin{array}{l}\text { Age at surgery } \\
\text { for AM (years) }\end{array}$} & \multirow[b]{2}{*}{ Gender } & \multicolumn{2}{|l|}{ Primary malignancy } & \multirow{2}{*}{$\begin{array}{l}\text { History of other } \\
\text { organ metastases }\end{array}$} \\
\hline & & & Pathological findings & Site & \\
\hline 1 & 74 & M & RCC (clear-cell carcinoma) & Right & None \\
\hline 2 & 47 & $\mathrm{~F}$ & Breast cancer (mammary carcinoma) & Left & Axillary lymph node \\
\hline 3 & 49 & $\mathrm{M}$ & RCC (clear-cell carcinoma) & Left & None \\
\hline 4 & 40 & M & Lung cancer (poorly differentiated adenocarcinoma) & Right & None \\
\hline 5 & 60 & $\mathrm{~F}$ & RCC (clear-cell carcinoma) & Left & Thyroid gland \\
\hline 6 & 74 & M & RCC (clear-cell carcinoma) & Left & Lung \\
\hline 7 & 77 & M & $\begin{array}{l}\text { Lung cancer } \\
\text { (mixture of large-cell carcinoma, spindle carcinoma, } \\
\text { giant-cell carcinoma and adenocarcinoma) }\end{array}$ & Left & None \\
\hline 8 & 56 & M & Lung cancer (large-cell carcinoma) & Right & None \\
\hline 9 & 58 & M & Lung cancer (well-differentiated adenocarcinoma) & Left & None \\
\hline
\end{tabular}

The present study analyzed 9 consecutive patients who underwent surgical resection of metastatic adrenal tumors in order to clarify the decision-making factors for LA or OA.

\section{Patients and methods}

From November 2003 to November 2006, 11 adrenalectomies were performed on 9 patients for adrenal metastasis (AM) for malignancies such as lung cancer, renal cell carcinoma (RCC) and breast cancer at Tokai University Hospital. All patients were treated after informed consent was obtained. Approval for the study was obtained from the institutional review board for the protection of human subjects at Tokai University School of Medicine. The patients included 7 male and 2 female individuals with a median age of $60 \pm 13$ years (range 40-77). A diagnosis of AM for the malignancies was suspected whenever a newly diagnosed adrenal mass occurred, characterized by basal computed tomography (CT) density superior to 10 Hounsfield units (HU), strong or heterogeneous vascular enhancement following contrast injection and/or increasing size in sequential imaging studies. Percutaneous adrenal biopsy was ruled out to prevent tumor seeding. All 9 patients underwent pre-operative staging with $\mathrm{CT}$, and there was no evidence of extra-AM. The approach to surgical management using LA or OA was determined on the basis of CT and/or magnetic resonance imaging (MRI). Patients in the LA group were placed in the lateral decubitus position. Four trocars were used. LA was performed with the greatest care to prevent tumor disruption. During the procedure, there was minimal handling of the tumor, and the adrenal gland was resected with its surrounding fat. Specimens were extracted intact within a bag. The patients were reviewed every 2 or 3 months by physical examination and systemic CT. The decision-making factors based on imaging concerning surgical management with LA or OA were analyzed according to the results of oncological outcome, imaging, intraoperative and pathohistological findings.

Statistical analyses were performed using commercially available software (SPSS ${ }^{\circledR}$ 15.0J, IL, USA). The paired $\mathrm{t}$-test was used for the statistical analysis of operative data. Distributions of biochemical disease-free survival times were calculated according to the Kaplan-Meier curves, and the logrank test was used to compare curves between the two groups. $\mathrm{P}<0.05$ was considered to be statistically significant.

\section{Results}

In the present study, 9 patients underwent 11 adrenalectomies (9 laparoscopic and 2 open procedures). Patient data and the characteristics of the primary malignancy are shown in Table I. Operative data and oncological outcomes of patients with AM are summarized in Table II. Non-small cell lung cancer (NSCLC) was the most common primary malignancy ( 5 adrenalectomies of 4 patients), followed by RCC (4 adrenalectomies of 4 patients) and breast cancer ( 2 adrenalectomies of 1 patient). There were 1 left, 6 right and 2 bilateral adrenalectomies, with a median tumor size of $3.9 \pm 1.4 \mathrm{~cm}$ (range 2.1-6.7). Of the adrenalectomies, 7 included metachronous metastases and 4 synchronous metastases.

The median tumor size for the LA group was $3.1 \pm 0.7 \mathrm{~cm}$ (range 2.1-4.3) and for the OA group, $6.1 \pm 0.8 \mathrm{~cm}$ (5.5 and $6.7 \mathrm{~cm})(\mathrm{p}=0.001)$. The operative time for the LA group was $127 \pm 42$ min (range 90-215) and for the OA group, $224 \pm 47 \min (190$ and $257 \mathrm{~min})(\mathrm{p}=0.018)$. Blood loss for the LA group was $49 \pm 63 \mathrm{~g}$ (range 3-207) and for the OA group, $340 \pm 10 \mathrm{~g}$ (333 and $347 \mathrm{~g})(\mathrm{p}<0.001)$. Among the patients with AM from RCC, 4 patients underwent contralateral LA $(n=3)$ and ipsilateral LA $(n=1)$. The median operative time for the contralateral group was $166 \pm 65 \mathrm{~min}$ (range 92-215) and for the ipsilateral case, $103 \mathrm{~min}$. Blood loss for the contralateral group was $163 \pm 62 \mathrm{~g}$ (range 92-207) and for the ipsilateral case, $17 \mathrm{~g}$. No complications were noted and no conversion of LA to OA occurred. All 9 adrenal tumors selected for LA were removed safely by LA without strong adhesion to the surrounding tissue. Two adrenal tumors removed by OA had strong adhesion to the surrounding tissue. In all cases, pathological examination confirmed the diagnosis of metastasis related to the primary malignancy. All of the patients had complete resection without capsular disruption and a negative margin in the pathological findings. Moreover, no port-site and local recurrence occurred. 


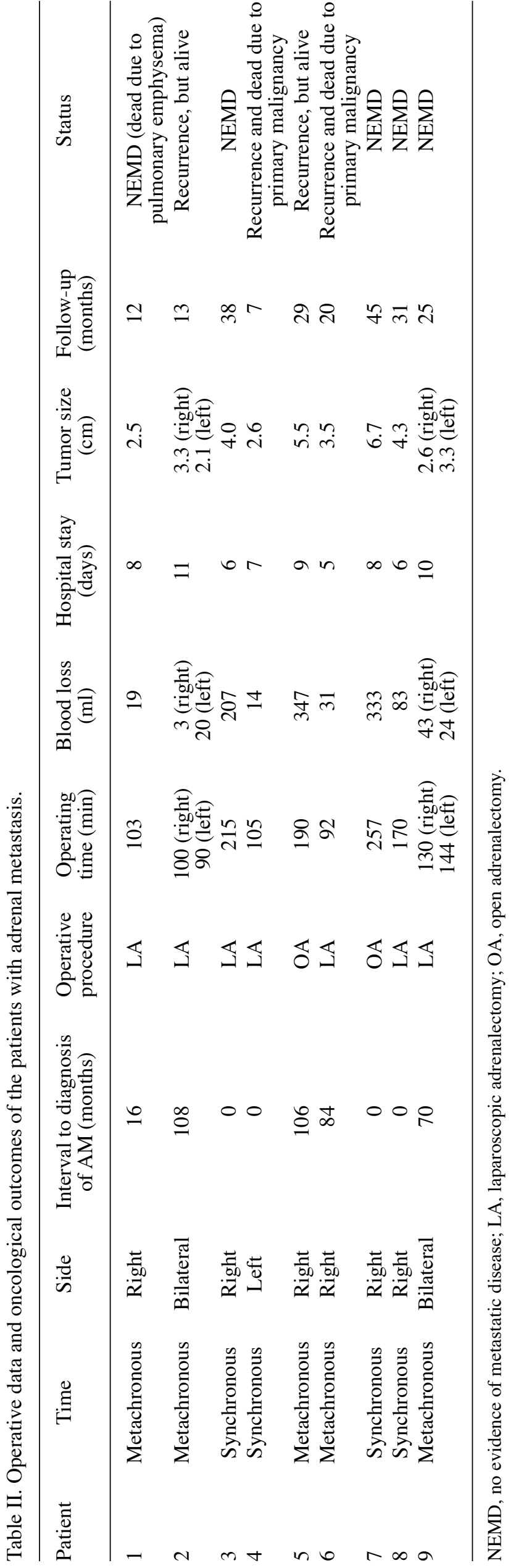

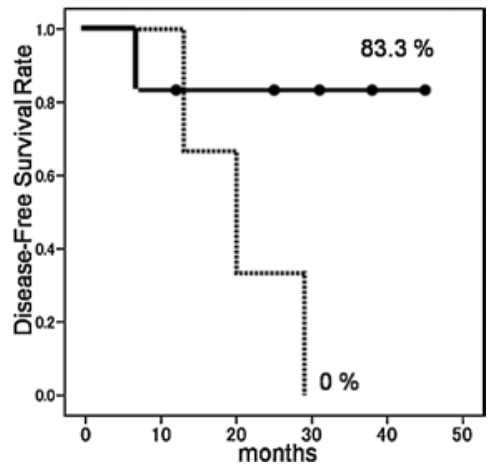

Figure 1. Disease-free survival rate for the group of patients with isolated AM (---) and AM with a history of other organ metastases (-).

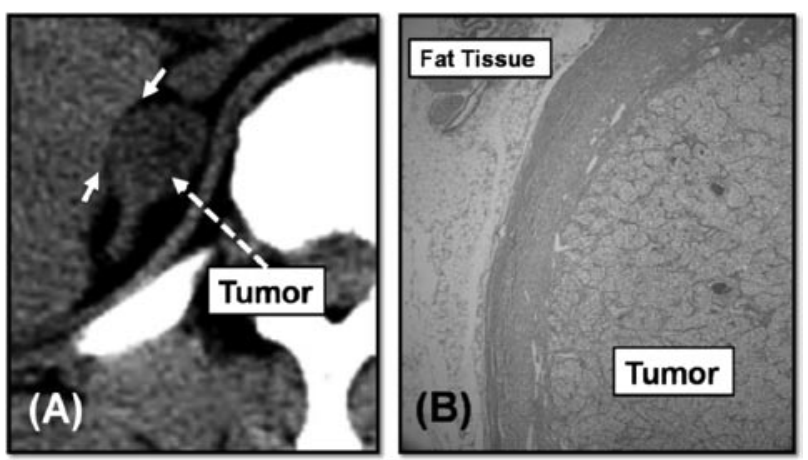

Figure 2. Patient 1. (A) Tumor size of the AM was $2.5 \mathrm{~cm}$. The tumor shape was round and smooth, and fatty tissue was detected around the tumor by CT $(\rightarrow)$. (B) According to the pathological findings, the adrenal tumor was clear-cell carcinoma, as well as the primary malignancy, and the border between the tumor and the surrounding fatty tissue was evident.

Pathological findings indicated fibrosis and the infiltration of inflammatory cells in the fatty tissue around the tumors removed by OA, while there was no fibrosis and infiltration of inflammatory cells in the fatty tissue surrounding the tumors removed by LA. The durations of the hospital stay after LA and OA were $8 \pm 2$ (range 5-11) and $9 \pm 0.5$ days (range 8-9), respectively $(\mathrm{p}=0.595)$. The observed follow-up term was $24 \pm 12$ months (range 12-45). No patients presented with local relapse or port-site metastasis. Median overall disease-free survival (MODFS) was $29 \pm 7$ months (range 7-38) and the disease-free survival rate (DFSR) was 55.6\%. DFSR for the LA group was $57 \%$ and for the OA group, $50 \%(\mathrm{p}=0.661)$. MODFS for the group with no history of other metastases was 39 \pm 6 months (range 7-45) and for the group with a history of other organ metastases, $21 \pm 5$ months (range 13-29). DFSR for the group with isolated AM was $83.3 \%$, and DFSR for those with a history of other organ metastases was $0 \%$ ( $\mathrm{p}=0.077$ ) (Fig. 1). MODFS for the metachronous group was $23 \pm 4$ months (range 13-29) and for the synchronous group, $30 \pm 7$ months (range 7-38). DFSR for the metachronous group was $40 \%$ and for the synchronous group, $75 \%(\mathrm{p}=0.435)$.

\section{Discussion}

LA has been acknowledged as the gold standard treatment for small benign secreting tumors, while it remains a subject of 


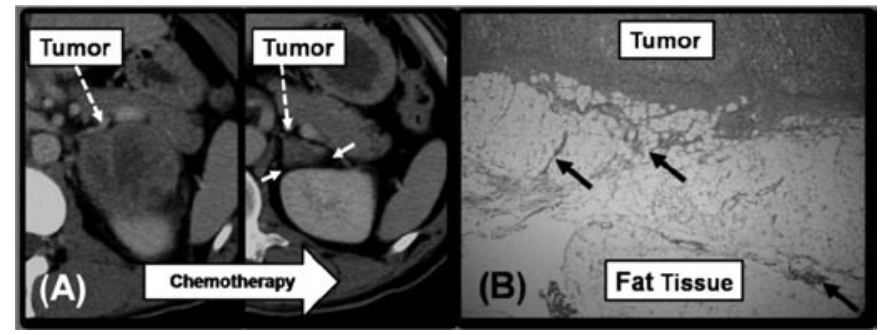

Figure 3. Patient 4. (A) Following adjuvant chemotherapy, the tumor size of the AM was reduced to $2.6 \mathrm{~cm}$, and the fatty tissue surrounding the tumor was detected by CT $(\rightarrow)$. (B) According to the pathological findings, the adrenal tumor was diagnosed as poorly differentiated adenocarcinoma as well as the primary malignancy, and fibrosis was evident in the fatty tissue around the tumor $(\rightarrow)$.

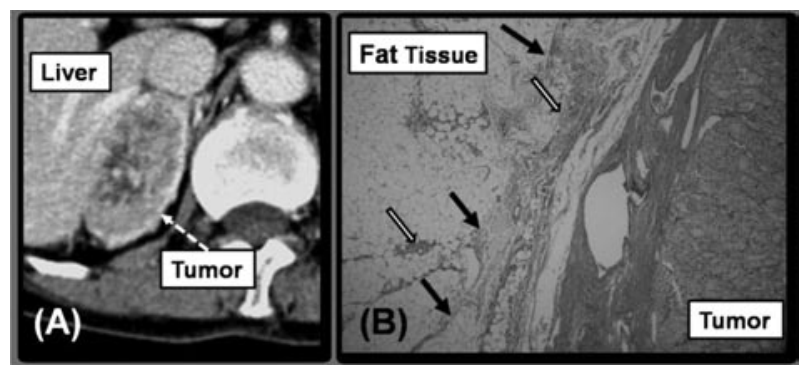

Figure 4. Patient 5. (A) The tumor size of the AM was $5.5 \mathrm{~cm}$. Fatty tissue around the tumor was not detected between the tumor and liver, and the contour of the tumor was irregular in CT. (B) According to the pathological findings, the adrenal tumor was diagnosed as clear-cell carcinoma, as well as the primary malignany, and fibrosis $(\rightarrow)$ and the infiltration of inflammatory cells $(\Rightarrow)$ were evident in the surrounding fatty tissue
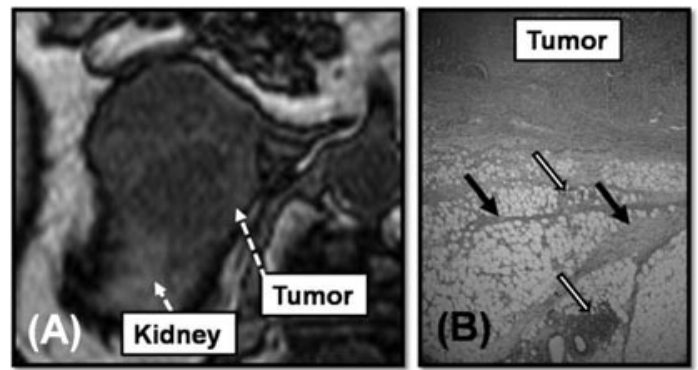

Figure 5. Patient 7. (A) The tumor size of the AM was $6.7 \mathrm{~mm}$. Fatty tissue around the tumor was not detected between the right kidney and tumor. The MRI showed the tumor to have an irregular contour and to be cystic (B) According to the pathological findings, the adrenal tumor was diagnosed as the primary malignancy, and fibrosis $(\rightarrow)$ and the infiltration of inflammatory cells $(\Rightarrow)$ were evident in the surrounding fatty tissue.

debate for large and potentially malignant adrenal tumors and AM. Long-term survival after resection of isolated adrenal metastatic tumors was first reported in 1982 by Twomey et al (3). The 2 patients in their study were disease-free for 6 and 14 years following resection of isolated adrenal metastases from large-cell lung cancer. Since then, a number of series, including several from the Memorial Sloan-Kettering Cancer Center, confirmed that when metastasis is isolated to the adrenal gland, adrenalectomy achieves long-term survival (2). These series showed that LA, compared to OA, resulted in less morbidity and achieved similar oncological outcomes. Evaluation of survival time points demonstrated that patients with synchronous metastasis had 73 and 38\% 1- and 3-year survival following adrenal resection, respectively, while patients with metachronous lesions had 86 and 46\% 1- and 3-year survival, respectively ( $p=0.62$ ). No significant difference in survival between patients treated with LA and those treated with OA $(p=0.43)$. Suzuki reported that survival ranged from 20 to 30 months after surgical removal of AM, compared to a mean survival of 6-8 months for patients whose AM was not resected (4).

In the present study, the 7 adrenalectomies of 6 patients were for isolated AM and the other 4 adrenalectomies were performed for 3 patients who had histories of other organ metastases. Adrenalectomy is a more effective treatment for isolated AM than for AM associated with a history of other organ metastases, as there were significant differences for DFSR in our study between the group of patients with isolated AM and patients with AM associated with a history of other organ metastases. However, this does not mean that adrenalectomy is not effective for patients with AM associated with a history of other organ metastases. Among the patients who had histories of other organ metastases, 2 adrenalectomies were performed for 2 patients who had metastasis from RCC and 2 adrenalectomies were perfomed for 1 patient who had metastasis from breast cancer. Certain patients with metastatic RCC may be treated with radical nephrectomy and resection of the metastatic lesions. A recent study found that metastasectomy was of clinical benefit across a range of prognostic groups and was independently associated with survival (5). Regarding AM from breast cancer, the optimal treatment is unclear, but early recognition and adrenalectomy of the tumor has potential survival benefits (6). For isolated AM in our study, only 1 patient (patient 4) relapsed in relation to the opposite adrenal gland at 11 months after LA. The primary malignancy was poorly differentiated adenocarcinoma of lung cancer. We considered that the case of isolated AM from high-grade malignancy had potent metastasis. Such a case requires careful follow-up.

We noted 4 patterns of pre-operative imaging and adrenalectomy for AM in this study. The first pattern was AM whose size was small with fatty tissue detected surrounding the tumor by imaging. The second pattern noted was the size of the AM reduced by chemotherapy. The third pattern was AM with a large size and irregular contour. The fourth pattern was AM of a large size and with a cystic component. A representative case of the first pattern was patient 1 . Patient 1 received radical nephrectomy for right RCC. AM was diagnosed at 16 months after radical nephrectomy. The tumor size of the AM was $2.5 \mathrm{~cm}$. The tumor shape was round and smooth and fatty tissue was detected surrounding the tumor by CT (Fig. 2A). We performed resection including the surrounding fat. Fatty tissue is required to detach the tumor and proximal organs. We performed LA for the tumor without any adhesion. According to the pathological findings, the adrenal tumor was diagnosed as clear-cell carcinoma, and as the primary malignancy, and the border between the tumor and the surrounding fatty tissue was evident (Fig. 2B).

The second pattern was exemplified by patient 4 . Patient 4 received video-assisted thoracic surgery (VATS) for right lung cancer. The pathological finding of the lung cancer indicated poorly differentiated adenocarcinoma. Lung cancer and left AM 
were diagnosed at a specific point in time with no evidence of extra-AM. Following adjuvant chemotherapy, the tumor size of AM was reduced to $2.6 \mathrm{~cm}$, and the fatty tissue surrounding the tumor was detected by CT (Fig. 3A). We performed LA for the tumor with slight adhesion. According to the pathological findings, the adrenal tumor was diagnosed as poorly differentiated adenocarcinoma and as the primary malignancy, and fibrosis was evident in the fatty tissue surrounding the tumor (Fig. 3B). The third pattern was noted in patient 5 who received radical nephrectomy for right RCC. The pathological finding of RCC indicated clear-cell carcinoma. AM was diagnosed 103 months after radical nephrectomy. The tumor size of AM was $5.5 \mathrm{~cm}$. Fatty tissue surrounding the tumor was not detected between the tumor and liver, and the contour of the tumor was irregular in CT (Fig. 4A). Detaching the tumor and liver was determined to be difficult and an OA was performed. During the surgical operation, we encountered strong adhesion between the tumor and the liver. According to the pathological findings, the adrenal tumor was diagnosed as clear-cell carcinoma, and as the primary malignancy. Fibrosis and the infiltration of inflammatory cells were evident in the fatty tissue between the tumor and the liver (Fig. 4B). Fibrosis and inflammatory cells play a role in adhesiogenesis (7). Therefore, the pathological findings of fibrosis and the infiltration of inflammatory cells in fatty tissue were in accordance with adhesion in the operative findings.

The fourth pattern was exemplified by patient 7 . This patient was diagnosed with left lung cancer and a right adrenal tumor, and VATS was performed for the left lung cancer. The pathological finding of lung cancer indicated a combination of large-cell carcinoma, spindle carcinoma, giant-cell carcinoma and adenocarcinoma. The tumor size of AM was $6.7 \mathrm{~cm}$. Fatty tissue surrounding the tumor was not detected between the right kidney and the tumor, and MRI revelaed the tumor to have an irregular contour and to be cystic (Fig. 5A). Detaching the tumor and kidney was determined to be difficult and a risk of perforating the cystic component was identified. We performed OA with nephrectomy as strong adhesion was detected between AM and the right kidney as expected. According to the pathological findings, the adrenal tumor was diagnosed as the primary malignancy, and fibrosis and the infiltration of inflammatory cells were evident in the fatty tissue between AM and the right kidney (Fig. 5B). The pathological findings were also in accordance with the operative findings for patient 5. We anticipated adhesion between the tumor and proximal organs from an imaging study. However, adhesion should be considered as in the case of patient 4. Fatty tissue between the tumor and proximal organs was detected after chemotherapy, but there was adhesion in the operative findings, and fibrosis was noted in the fatty tissue around the tumor in the pathological findings.

In the present study, no port-site and local recurrence occurred. Port-site recurrence is reported rarely $(8,9)$. Local recurrence and port-site metastases are associated with four factors: natural tumor behavior, local wound factors, immune and stress responses, and laparoscopic-related factors such as surgical manipulation (10). Great care must be taken in the surgical manipulation to prevent dissemination. We performed adrenalectomy with minimal handling of the tumor. The adrenal gland was resected with its surrounding fat to prevent tumor disruption and there was no port-site and local recurrence; minimal handling of the tumor and tumor resection with its surrounding fat are crucial in the prevention of port-site and local recurrence. Furthermore, the ultrasonically activated scalpel or ultrasonic endoaspirator should be carefully handled so that it does not touch the tumor surface as this may create a risk of tumor cell dissemination.

Various factors aid in the decision-making process when determining whether the most appropriate approach is LA or OA. When imaging did not detect fatty tissue around the tumor, we predicted an irregular contour and adhesion. When fat tissue is not clearly detected around the tumor, MRI may be more effective due to its high ability to detect adipose. Detecting adipose may help avoid incomplete resection, which often means converting LA to OA (8). Moreover, it was reported that the volume of blood loss from large adrenal tumors is significantly greater than that from small tumors (4). Therefore, a large AM has a risk of extensive blood loss. A tumor with a cystic component that is difficult to detach due to adhesion presents a risk of perforation and dissemination.

Moreover, in the case of AM from RCC, a contralateral case involves a longer operative time and more blood loss than an ipsilateral case. Therefore, contralateral AM from RCC is difficult to treat with adrenalectomy. Thus, decision making for the appropriate surgical management, whether to apply OA or LA, is crucial.

In conclusion, LA is a less invasive treatment than OA for AM. However, for complete resection, OA should be selected for cases where resection by LA is difficult. Therefore, in decision making regarding the appropriate surgical management with LA or OA, it is crucial to closely assess pre-operative imaging. Imaging features supporting OA include: no detection of fatty tissue between the tumor and proximal organs, tumors with an irregular contour, large tumors and tumors with a cystic component.

\section{References}

1. Gagner M, Lacroix A and Bolté E: Laparoscopic adrenalectomy in Cushing's syndrome and pheochromocytoma. N Engl J Med 327: 1033, 1992.

2. Strong V, D'Angelica M, Tang L, et al: Laparoscopic adrenalectomy for isolated adrenal metastasis. Ann Surg Oncol 14: 3392-3400, 2007.

3. Twomey P, Montgomery C and Clark O: Successful treatment of adrenal metastases from large-cell carcinoma of the lung. JAMA 248: 581-583, 1982.

4. Suzuki H: Laparoscopic adrenalectomy for adrenal carcinoma and metastases. Curr Opin Urol 16: 47-53, 2006.

5. Eggener E, Yossepowitch O, Kundu S, et al: Risk score and metastasectomy independently impact prognosis of patients with recurrent renal cell carcinoma. J Urol 180: 873-878, 2008.

6. Liu J, Shen P, Wang F, et al: Solitary adrenal metastasis from invasive ductal breast cancer: an uncommon finding. World $\mathrm{J}$ Surg Oncol 28: 7-10, 2010.

7. Liakakos T, Thomakos N, Fine M, et al: Peritoneal adhesions: etiology, pathophysiology, and clinical significance. Recent advances in prevention and management. Dig Surg 18: 260-273, 2001.

8. Sebag F, Calzolari F, Harding J, et al: Isolated adrenal metastasis: the role of laparoscopic surgery. World J Surg 30: 888-892, 2006.

9. Weyhe D, Belyaev O, Skawran S, et al: A case of port-site recurrence after laparoscopic adrenalectomy for solitary adrenal metastasis. Surg Laparosc Endosc Percutan Tech 17: 218-220, 2007.

10. Tanaka K, Hara I, Takenaka A, et al: Incidence of local and port site recurrence of urologic cancer after laparoscopic surgery. Urology 71: 728-734, 2008. 
